\title{
Evaluation of Thin Layer Agar 7H11 for Direct Susceptibility Testing of Mycobacterium Tuberculosis Complex against Second Line Anti Tuberculosis Drugs on Smear Positive Specimens
}

\author{
Ashfaq Hussain1, Gohar Zaman1, Luqman Satti1, Aamer Ikram², \\ Adeel Hussain Gardezi ${ }^{1}$ and Muhammad Tahir Khadim 1
}

\begin{abstract}
Objective: To evaluate performance of thin layer agar (TLA) 7H11 method for detection of ofloxacin (OFX) and kanamycin (KM) resistance in smear positive clinical specimens of patients with tuberculosis comparing the results with gold standard MGIT 960 system.

Study Design: Cross-sectional validation study.

Place and Duration of Study: Department of Microbiology, Armed Forces Institute of Pathology (AFIP), Rawalpindi, from April to September 2017.

Methodology: Acid fast bacilli (AFB) smear positive specimens submitted at the study place, were inoculated on TLA 7H11 agar. Growth was examined along with susceptibility of OFX and KM and compared with gold standard MGIT 960 system. Results: One hundred and sixty specimens were evaluated. Sensitivity and specificity of TLA for OFX was found to be $100 \%$ and $99.3 \%$, respectively; and PPV and NPV was found to be $90.9 \%$ and $100 \%$, respectively. Overall diagnostic accuracy was $99.38 \%$. Sensitivity and specificity of TLA for KM was found to be $80 \%$ and $100 \%$, respectively. PPV and NPV was found to be $100 \%$ and $99.36 \%$, respectively. Overall diagnostic accuracy was $99.38 \%$.

Conclusion: Thin layer agar is reliable, easy to perform and cost effective technique not only for rapid detection of MTB but also for drug susceptibility (DST) of second line anti TB agents. It is a suitable alternative to culture on LJ medium and can also be alternative to MGIT 960 system in resource-poor settings.
\end{abstract}

Key Words: Drug susceptibility testing, Mycobacteria growth indicator tube, Thin-layer agar, Mycobacterium tuberculosis complex.

\section{INTRODUCTION}

Mycobacterium tuberculosis complex (MTB) is the agent of disease, i.e. tuberculosis (TB), which claims more than 1.6 million lives annually. ${ }^{1}$ There were 10.4 million new cases of TB worldwide in 2016. All age groups were affected but $90 \%$ cases were adults. ${ }^{2}$ According to WHO Global TB Report $2017,56 \%$ of all new TB cases were found to be from top five high TB-burden countries, which also include Pakistan. Multi-drug resistant-TB (MDR-TB) was found in $4.1 \%$ new cases and $19 \%$ patients were those who were already treated for TB. 3

Resistance of MTB to anti-tuberculous (anti-TB) drugs remains a major challenge in treatment of tuberculosis. MDR-TB is defined as in vitro resistance to isoniazid (INH) and rifampicin (RIF) among MTB isolates. There may or may not be resistance to other first line anti-TB

1 Department of Microbiology, Armed Forces Institute of Pathology, Rawalpindi, Pakistan

2 Department of Microbiology, National Institute of Health, Islamabad, Pakistan

Correspondence: Dr. Muhammad Luqman Satti, Department of Microbiology, Armed Forces Institute of Pathology (AFIP),

Rawalpindi, Pakistan

E-mail: luqmansatti@hotmail.com

Received: September 08, 2018; Accepted: December 18, 2018 drugs. Extensively drug resistant TB (XDR-TB) is defined as MDR-TB plus in vitro resistance to fluoroquinolones; and one of the injectable drugs, i.e. kanamycin, amikacin and capreomycin. ${ }^{4}$ Timely and accurate drug susceptibility testing (DST) is one of the key factors in reducing TB-related morbidity and mortality. A large number of hospitals and laboratories are relying on sputum smear microscopy by Ziehl- Neelson staining for TB diagnosis without performing DST.5-9 Conventional egg-based culture medium, Lowenstein-Jensen (LJ) medium, remains most commonly used method for DST; but it takes too long, around 8-10 weeks for susceptibility testing. Liquid based culture, mycobacterial growth indicator tube (MGIT) is rapid method which takes around 21 to 28 days for DST. It is semi-automated system which requires costly equipment and more prone to contamination as compared to solid culture media. $4,10,11$

There are various molecular methods as well, for DST of MTB, which have got high sensitivity and specificity, require less training, less time consuming, and comparatively simple. Xpert MTB/RIF is automated method which can only detect resistance of RIF in less than two hours. Line probe assays have been evaluated for MTB drug resistance detection in respiratory specimens. MTBDRs/ is only molecular method available for detection of resistance to second line anti-TB agents. 
These methods are costly, require infrastructure, and can test susceptibility of limited number of anti-TB drugs; so molecular methods cannot eliminate requirement of culture-based methods. $4,9,12-13$

Therefore, there is an urgent need for the development of a simple, rapid but cost effective technique for the detection of MDR and XDR-TB in developing countries.6,11,14 To reduce the time of diagnosis and DST, several non-commercial methods such as calorimetric redox indicator (CRI) method, microscopic observation drug susceptibility (MODS) assay, nitrate reductase assay (NRA) and thin-layer agar (TLA) have been developed. $5,9,15-17$

TLA containing Middlebrooks $7 \mathrm{H} 11$ medium is a costeffective method, which does not require any sophisticated equipment. It detects growth of MTB within two weeks, based on its characteristic colony morphology (observed under microscope) and also gives DST. It has demonstrated good performance for $\mathrm{INH}$ and RIF.5-6, 10,12,14

The objective of this study was to evaluate TLA method for determing sensitivity of second-line drugs OFX and $\mathrm{KM}$ in AFB smear positive clinical specimens and compare it with the MGIT 960 system.

\section{METHODOLOGY}

This cross-sectional validation study was carried out at the Department of Microbiology, Armed Forces Institute of Pathology, Rawalpindi, Pakistan. It is a tertiary care reference laboratory with BSL-3 facilities for MTB culture and sensitivity. Permission was obtained from the Institutional Ethical Committee.

All the smear-positive specimens (pulmonary and extrapulmonary) were included. No discrimination was made on the basis of age and gender. Repeat samples of same patient and samples from patients already on anti-tuberculosis agents were excluded. Endobronchial (EB) washings, sputum and bronchoalveolar lavage (BAL) were included in pulmonary specimens while pus, tissues and pleural fluid were included in extrapulmonary specimens. All pulmonary specimens and pus specimens were digested and decontaminated with final concentration of $1.5 \% \mathrm{~N}$-acetyl-L-cysteine- $\mathrm{NaOH}$. After centrifugation for 20 minutes at $3000 \mathrm{~g}$, pellet sediment was resuspended phosphate buffer (PH 6.8) to achieve final volume of $3 \mathrm{ml}$. Large pieces of tissue specimens were minced. Material was then homogenised with sterile saline. Suspension was then processed as pulmonary specimens. Urine specimen was centrifuged at $3600 \times \mathrm{g}$ for 30 minutes. Two $\mathrm{ml}$ of sediment was processed as pulmonary specimens. Pleural fluids were centrifuged at $3000 \mathrm{x} \mathrm{g}$ for 15 minutes. Sediment was used for inoculation onto media. No decontamination procedure was done for sterile fluids. All the processed specimens were inoculated in
BACTEC MGIT 960 system as well as on TLA. M. tuberculosis H37Ra (ATCC 25177) was used as a sensitive strain and institutional XDR strain was used as resistant strain for quality control (QC) testing in DST.

Inoculation on MGIT 960 was done as per the manufacturer's instructions. Tubes were incubated inside the system at $37^{\circ} \mathrm{C}$ after scanning the barcode. Tubes were incubated for a period of six weeks before being labelled as negative. After being flashed positive on system, tube were removed from system, $\mathrm{ZN}$ staining was done for confirmation of presence of AFB and sub-culturing on blood agar was done to check for contaminants. To differentiate MTB complex from non-tuberculous mycobacteria (NTM), PNB test was performed. After confirmation of MTB complex by ZN staining and PNB test, DST for OFX and KM was performed using three MGIT tubes: one for the control, and one each for the agents using standard laboratory protocol. All negative tubes with visible turbidity were subjected to $Z N$ staining at the end to find out any false negative results.

In TLA method, Middlebrook $7 \mathrm{H} 11$ agar was used. $5 \mathrm{ml}$ of $7 \mathrm{H} 11$ agar was poured per compartment into a quadrant petri dish after addition of $10 \%$ OADC (oleic acid, albumin, dextrose, and catalase) and antibiotics mixture PANTA (Polymyxin-B, Amphotericin-B, Nalidixic acid, Trimethoprim and Azlocillin). One compartment was growth control (GC), second had addition of $2.0 \mu \mathrm{g} / \mathrm{ml}$ OFX, the third compartment had $6.0 \mu \mathrm{g} / \mathrm{ml} \mathrm{KM}$ and the last one with $500 \mu \mathrm{g} / \mathrm{ml}$ of PNB. 7,12 Ten $\mu \mathrm{l}$ of processed specimen was added to each compartment. Plates were sealed with adhesive tape leaving space of $1-2 \mathrm{~cm}$ and was incubated at $37^{\circ} \mathrm{C}$ in $5 \% \mathrm{CO}_{2}$ incubator for 6 weeks. Plates were checked after 24 hours for contamination and examined for micro-colonies twice weekly by using conventional microscope with low power objective $(10 \mathrm{x})$. Time for detection of micro-colonies was noted. Growth in GC compartment represented growth of mycobacteria species; and growth in PNB compartment suggested NTM and were excluded from study. Number of colonies on the growth control compartment were counted. Resistance was defined as more than $1 \%$ of the growth on drug containing compartment as compared to number of colonies on drug-free GC compartment. Similarly, susceptibility was defined as no growth or less than $1 \%$ of the growth on drug containing compartment as compared to number of colonies on drug-free compartment. Fungal or bacterial contamination was recognised by rapid overgrowth on plates. 5,12

The data was entered in SPSS version 24 software for statistical analysis. Descriptive statistics was calculated for both qualitative and quantitative variables. For quantitative variables like age, mean and standard deviation (SD) were calculated. For qualitative variables like gender, frequency and percentage were calculated. A 2x2 table was made to calculate sensitivity, specificity, NPV, PPV and diagnostic accuracy of TLA for both OFX 
and KM. Chi-square test was used to find association between qualitative variables and $p$-value of $<0.05$ was considered significant. Sensitivity was taken as representing the ability of a method to detect true resistance; and specificity to indicate the ability of a method to detect true sensitivity of an isolate.

\section{RESULTS}

A total of 178 patient's specimens were considered in the study. Nine specimens yielded growth of NTM, seven specimens were contaminated and two specimens did not yield growth on GC compartment of the plate. These 18 specimens were excluded from study and 160 specimens were included in the study. Out of 160 patients, 98 (61.3\%) were males and 62 (38.8\%) were females. Mean age of patients was $44.51 \pm 33.28$ years, minimum age of 11 years and maximum age of 70 years. One hundred and four patients (65\%) were from outpatient department (OPD) and 56 patients (35\%) were indoor. Majority of specimens received were endobronchial (EB) washings $(74,46.3 \%)$ followed by sputum $(59,36.9 \%)$, bronchoalveolar lavage $(11,6.9 \%)$, pus $(6,3.8 \%)$, pleural fluid $(5,3.1 \%)$, tissues $(4,2.5 \%)$ and urine (1, 0.6\%). Out of 160 specimens, $99(61.9 \%)$ had mycobacterial index of $1+, 41(25.6 \%) 2+, 10(6.3 \%)$ $3+$ and $10(6.3 \%) 4+$ index. Specimens with $1+$ index yielded growth of MTB on TLA between 9 days to 24 days as shown in Table I. Overall growth of MTB on TLA showed a large variation from 4 to 24 days (mean 13.7 days) depending on mycobacterial index (Table I). Eleven specimens (6.9\%) were MDR- TB i.e. resistant to both INH and RIF, 25 specimens (15.6\%) were monoresistant to INH. Sensitivity and specificity of TLA for OFX was found to be $100 \%$ and $99.3 \%$, respectively as shown in Table II. Overall diagnostic accuracy was $99.38 \%$ with ROC curve value of 1.00 and positive and negative likelihood ratio of 150 and zero, respectively. Sensitivity and specificity of TLA for KM was found to be $80 \%$ and $100 \%$, respectively (Table II). Overall diagnostic

Table I: Relationship of Mycobacterial index with Time to detection on $\operatorname{TLA}(n=160)$.

\begin{tabular}{l|c|c|c}
\hline Mycobacterial index & Frequency & Percentage & Time to detection on TLA \\
\hline $1+$ & 99 & $61.9 \%$ & $9-24$ days (Mean 15 days) \\
$2+$ & 41 & $25.6 \%$ & $9-18$ days(Mean 13 days) \\
$3+$ & 10 & $6.3 \%$ & $7-12$ days (Mean 10 days) \\
$4+$ & 10 & $6.3 \%$ & $4-8$ days (Mean 6 days) \\
\hline Total specimens & 160 & $100 \%$ & $4-24$ days (Mean 13.7 days) \\
\hline
\end{tabular}

accuracy was $99.38 \%$ with ROC curve value of 0.90 and positive and negative likelihood ratio of zero and 0.2 , respectively.

\section{DISCUSSION}

Tuberculosis (TB) remains a deadly communicable disease despite all advancements in medical sciences. 16 MDR-TB and XDR-TB is a serious public and social health concern that has gained global focus. One of the biggest challenges is emergence of XDR-TB. It is estimated that $9.7 \%$ of MDR-TB cases develop XDR-TB, which is actually more worrisome.18,19 Success rate in XDR-TB treatment is significantly low. Treatment failure not only leads to transmission of infection but also leads to chronic lung disease. Early and accurate diagnosis is the key to prompt management of TB cases, prevention of transmission, prevention of MDR-TB and XDR-TB incidence and better clinical outcome.5,16

In this study, growth of MTB on TLA showed large variation from 4 to 24 days (mean 13.7days) depending on mycobacterial index. A study done by Satti et al. showed that mean time to detection of mycobacteria on TLA for smear-positive cases, was 10.1 (4-22) days; while for smear-negative cases, it was 15.8 (12-28) days on TLA. 20 Another study done by Iftikhar et al. showed that in TLA method, mean drug susceptibility turnaround time shortened to 10.2 days. ${ }^{21}$ Results were quite comparable to these studies. In this study, time to detection was longer than these studies. One possible reason could be that most of our cases $(87.5 \%)$ had mycobacterial index of $1+$ and $2+$.

In this study, only 10 out of 160 cases (6.25\%) were OFX resistant and 150 cases (93.25\%) were OFX sensitive; while only 5 cases $(3.1 \%)$ were $\mathrm{KM}$ resistant and 155 cases $(96.9 \%)$ were $\mathrm{KM}$ sensitive (Table II). A study done by Ardizzoni et al. showed 2.1\% OFX resistance and $3.9 \% \mathrm{KM}$ resistance. ${ }^{12}$ Another study done by Iftikhar et al. showed $40 \%$ OFX resistance and $6 \% \mathrm{KM}$ resistance. 21 The present $\mathrm{KM}$ resistance data is comparable with both local and international studies, but OFX resistance is quite low as compared to local studies. 21

In this study, sensitivity and specificity of TLA for OFX was found to be $100 \%$ and $99.3 \%$, respectively, PPV and NPV was found to be $90.9 \%$ and $100 \%$, respectively. Overall diagnostic accuracy was $99.38 \%$. Sensitivity and specificity of TLA for KM was found to be $80 \%$ and $100 \%$, respectively. Overall diagnostic accuracy was $99.38 \%$.

Table II: Comparison of results of MGIT 960 with results of sensitivity on TLA $(n=160)$.

\begin{tabular}{l|c|cc|c|c|c|c|c}
\hline Drugs & TLA & \multicolumn{2}{|c|}{ MGIT } & \multicolumn{3}{|c}{ TLA $\%$} \\
\hline OFX & & $\mathrm{R}$ & $\mathrm{S}$ & Sensitivity & Specificity & PPV & NPV & DA \\
& $\mathrm{R}$ & 10 & 1 & $100 \%$ & $99.3 \%$ & $90.9 \%$ & $100 \%$ & $99.3 \%$ \\
\hline $\mathrm{KM}$ & $\mathrm{S}$ & 0 & 149 & & & & 9 \\
& $\mathrm{R}$ & 4 & 0 & $80 \%$ & $100 \%$ & $100 \%$ & $99.36 \%$ & $99.3 \%$ \\
\hline
\end{tabular}

PPV = Positive predictive value; $N P V=$ Negative predictive value; $\quad D A=$ Diagnostic accuracy 
Previously, a study was conducted at our institute on diagnostic accuracy of TLA against first-line anti-TB drugs isoniazid (INH) and rifampicin (RIF). The study showed sensitivity and specificity of TLA for INH to be $71 \%$ and $72 \%$, respectively; while sensitivity and specificity of TLA for RIF was found to be $69 \%$ and $64 \%$, respectively. ${ }^{5}$

According to a study done by Martin et al., sensitivity and specificity of TLA for OFX was $100 \%$. Overall diagnostic accuracy was $100 \%$. Sensitivity and specificity of TLA for KM was found to be $100 \%$ and $98 \%$, respectively; while overall diagnostic accuracy was $99.3 \% .{ }^{7}$ In another study done by Ardizzoni et al., sensitivity and specificity of TLA for OFX was $100 \%$. Sensitivity and specificity of TLA for KM was found to be $78.9 \%$ and $100 \%$, respectively. 12 A local study done by Iftikhar et al. revealed sensitivity and specificity of TLA for OFX $92.1 \%$ and $96.1 \%$, respectively. Overall diagnostic accuracy was $93.75 \%$. Sensitivity and specificity of TLA for KM was found to be $100 \%$, while overall diagnostic accuracy was $100 \% .{ }^{21}$ Diagnostic accuracy for OFX and KM in this study was consistent with local and international studies.

There are two main limitations of TLA. First, it needs staff to be trained for microscopic detection of MTB, and microscopy could be time consuming in laboratories with heavy workload. Second, studies have shown that results of TLA are convincing on smear-positive cases only.

\section{CONCLUSION}

Thin layer agar is a reliable, easy to perform, and costeffective technique not only for rapid detection of MTB; but also for direct DST of anti-TB drugs. It is a suitable alternative to culture on LJ medium and can also be alternative to MGIT in poor resource settings. However, more studies on a large scale are needed to know its exact usefulness and accuracy.

\section{REFERENCES}

1. Cox H, Nicol MP. Tuberculosis eradication: Renewed commitment and global investment required. Lancet Infect Dis 2018; 18:228-9.

2. Floyd K, Glaziou P, Zumla A, Raviglione M. The global tuberculosis epidemic and progress in care, prevention, and research: An overview in year 3 of the end TB era. Lancet Respir Med 2018; 6:299-314.

3. WHO Global Tuberculosis Report 2017. World Health Organization, Geneva; 2017.

4. Oommen S, Banaji N. Laboratory diagnosis of tuberculosis: Advances in technology and drug susceptibility testing. Indian J Med Microbiol 2017; 35:323-31.

5. Hussain A, Mirza IA, Satti L, Ikram A, Ali S, Khan IU. Comparison of thin layer agar $7 \mathrm{H} 11$ and MGIT 960 for the detection of rifampicin and isoniazid resistance in mycobacterium tuberculosis complex. Infect Dis J 2012; 453.

6. Alam HB, Miah MR, Kamal SM, Roy CK, Saleh AA. Rapid drug susceptibility testing for mycobacterium tuberculosis in thin layer agar media. Bangladesh J Med Microbiol 2013; 7:2-6.
7. Martin A, Paasch F, Von Groll A, Fissette K, Almeida P, Varaine F, et al. Thin-layer agar for detection of resistance to rifampicin, ofloxacin and kanamycin in mycobacterium tuberculosis isolates. Int J Tuberc Lung Dis 2009; 13:1301-4.

8. Tayyab N, Zaman G, Satti L, Ikram A, Gardezi AH, Khadim MT. Direct susceptibility testing on MGIT 960 TB System: A rapid method for detection of drug resistant tuberculosis. $J$ Coll Physicians Surg Pak 2018; 28:590-3.

9. Wilson ML. Rapid diagnosis of mycobacterium tuberculosis infection and drug susceptibility testing. Arch Pathol Lab Med 2013; 137:812-9.

10. Battaglioli T, Rintiswati N, Martin A, Palupi KR, Bernaerts G, Dwihardiani B et al. Comparative performance of thin layer agar and Löwenstein-Jensen culture for diagnosis of tuberculosis. Clin Microbiol Infect 2013; 19:E502-8.

11. Satti L, Ikram A. Tuberculosis: Advancements in diagnosis and drug susceptibility testing. Gomal J Med Sci 2011; 9:137-9.

12. Ardizzoni E, Mulders W, Kotrikadze T, Aspindzelashvili R, Goginashvili L, Pangtey $\mathrm{H}$, et al. The thin-layer agar method for direct phenotypic detection of multi- and extensively drugresistant tuberculosis. Int J Tuberc Lung Dis 2015; 19:1547-52.

13. Saeed M, Ahmad M, Iram S, Riaz S, Akhtar M, Aslam M. GeneXpert technology: A breakthrough for the diagnosis of tuberculous pericarditis and pleuritis in less than 2 hours. Saudi Med J 2017; 38:699.

14. Hernández-Sarmiento JM, Martínez-Negrete MA, CastrillónVelilla DM, Mejía-Espinosa SA, Mejía-Mesa GI, ZapataFernández EM et al. Thin layer agar represents a cost-effective alternative for the rapid diagnosis of multi-drug resistant tuberculosis. Rev Salud Publica (Bogota) 2014; 16:101-13.

15. World Health Organization. Noncommercial culture and drugsusceptibility testing methods for screening patients at risk for multidrug-resistant tuberculosis: Policy statement World Health Organization 2011.

16. Ghafoor T, Ikram A, Abbassi SA, Mirza IA, Hussain A, Ullah Khan I, et al. Antimicrobial sensitivity pattern of clinical isolates of mycobacterium tuberculosis: A retrospective study from a reference laboratory in Pakistan. J Virol Microbiol 2014; 2014:1-6.

17. Boum Y, Orikiriza P, Rojas-Ponce G, Riera-Montes M, Atwine D, Nansumba $M$, et al. Use of colorimetric culture methods for detection of mycobacterium tuberculosis complex isolates from sputum samples in resource-limited settings. J Clin Microbiol 2013; 51:2273-9.

18. Zhang Q, Wu Z, Zhang Z, Sha W, Shen X, Xiao H. Efficacy and effect of free treatment on multidrug resistant tuberculosis. Exp Ther Med 2016; 11:777-82.

19. Hofman S, Segers MM, Ghimire S, Bolhuis MS, Sturkenboom MG, Van Soolingen $\mathrm{D}$, et al. Emerging drugs and alternative possibilities in the treatment of tuberculosis. Expert Opin Emerg Drugs 2016; 21:103-16.

20. Satti L, Ikram A, Abbasi S, Malik N, Mirza IA, Martin A. Evaluation of thin-layer agar $7 \mathrm{H} 11$ for the isolation of mycobacterium tuberculosis complex. Int J Tuberc Lung Dis 2010; 14:1354-6.

21. Iftikhar I, Irfan S, Farooqi J, Azizullah Z, Hasan R. Rapid detection of in vitro antituberculous drug resistance among smear-positive respiratory samples using microcolony detection-based direct drug susceptibility testing method. Int $J$ Mycobacteriol 2017; 6:117. 